\title{
Temporary Filtering Bleb Failure Induced by Anterior Chamber Sulfur Hexafluoride Gas: A Complication after Descemet Membrane Endothelial Keratoplasty
}

\author{
Philip Enders Georgia Avgitidou Ludwig M. Heindl \\ Thomas S. Dietlein Claus Cursiefen \\ Department of Ophthalmology, University Hospital of Cologne, Cologne, Germany
}

\section{Keywords}

Descemet membrane endothelial keratoplasty - Sulfur hexafluoride gas tamponade .

Filtration bleb · Glaucoma · Postoperative intraocular pressure peak

\begin{abstract}
Herein, we report two clinical cases with acute temporary filtering bleb obstruction by gas tamponade after Descemet membrane endothelial keratoplasty (DMEK) surgery and postoperative intraocular pressure (IOP) peaks. Both patients underwent uncomplicated DMEK surgery with $20 \%$ sulfur hexafluoride $\left(\mathrm{SF}_{6}\right)$ anterior chamber tamponade and had previous trabeculectomy for glaucoma. Prior to surgery, both patients showed patent bleb function with low to normal IOP without antiglaucomatous medication. After uneventful DMEK surgery, both patients showed postoperative IOP peaks of up to $50 \mathrm{~mm} \mathrm{Hg}$ despite patent inferior iridotomy and no sign of a pupillary block. In both cases, $\mathrm{SF}_{6}$ gas bubbles could be visualized obstructing the bleb. Both patients were treated with IOP-lowering agents topically as well as systemically. In addition, anterior chamber paracenteses were performed to reduce the $\mathrm{SF}_{6}$ volume within the anterior chamber. Under this treatment, IOP normalized within the first $18 \mathrm{~h}$ after surgery. We hypothesize that the $\mathrm{SF}_{6}$ gas tamponade from the anterior chamber migrates into the ostium and below the bleb, leading to an acute temporary insufficiency of bleb function and to
\end{abstract}


a consecutive IOP peak after surgery. In contrast to a pupillary block, this mechanism cannot be antagonized by preoperative iridotomy and needs to be taken into account for every glaucoma patient with functional bleb undergoing DMEK surgery.

\section{Introduction}

Anterior chamber tamponade with air or sulfur hexafluoride $\left(\mathrm{SF}_{6}\right)$ gas plays an essential role in Descemet membrane endothelial keratoplasty (DMEK) surgery [1]. This posterior lamellar keratoplasty technique is performed to treat endothelial disorders of the cornea by corneal transplantation [2-4]. At the end of surgery, air or $\mathrm{SF}_{6}$ gas with a concentration of $20 \%$ are injected into the anterior chamber to keep the thin transplant lamella in place and to foster graft attachment to the recipient's cornea [1]. Early graft detachment is a frequent postoperative complication [5, 6]. Divergent opinions have been expressed amongst DMEK surgeons on how long the gas bubble should be retained in the eye [7-10]. In order to prevent a pupillary block by the gas bubble, standard operating procedures include the preoperative application of YAG laser iridotomy or perioperative surgical iridectomy in the lower peripheral iris. The standard of care treatment after DMEK surgery includes topical application of dexamethasone every hour as well as antibiotics and miotics three times a day [11].

In glaucoma management, trabeculectomy is a long-established surgical approach to lower intraocular pressure (IOP). After successful surgery, aqueous humor is redirected to flow through an incision of the iris and sclera to form a subconjunctival bleb [12-15].

With presence of air or $\mathrm{SF}_{6}$ tamponade, a pupillary block can lead to an early postoperative IOP peak [6]. To prevent this complication after DMEK surgery, the standard operating procedures include performing an inferior iridotomy using the Nd:YAG laser. Other reasons for an acute postoperative IOP peak are bleeding to the anterior chamber or retained viscoelastic.

Here, we report on an additional reason for postoperative IOP peaking after DMEK in patients having previously undergone filtering bleb surgery. The acute temporary obstruction of a functional bleb by $\mathrm{SF}_{6}$ gas can also lead to a significant increase in IOP after surgery. This complication needs to be anticipated in glaucoma patients with functional bleb undergoing DMEK surgery. Patients at risk should be monitored very closely to detect and treat any IOP peak within the early postoperative phase.

\section{Case 1}

In November 2018, an 80-year-old female patient received a pseudophakic DMEK procedure in her right eye to treat corneal edema due to Fuchs endothelial dystrophy. The patient had received trabeculectomy for primary open-angle glaucoma 5 years before. The diagnosis of primary open-angle glaucoma dated back to 1987. Preoperatively, the right eye scheduled for DMEK surgery had a best corrected visual acuity (BCVA) of $0.8 \operatorname{logMAR}$ and an IOP of $10 \mathrm{~mm} \mathrm{Hg}$. On admission to surgery, the patient had no topical or systemic IOP-lowering medication. On slit-lamp examination, the trabeculectomy bleb was well prominent and functional.

Six hours after uneventful DMEK surgery - performed by an experienced surgeon (C.C.) in a standardized fashion [16] - routine IOP measurement showed a normal IOP. Four hours later, the patient complained about increasing pain in the right eye. IOP was measured at 
$50 \mathrm{~mm} \mathrm{Hg}$ in the right eye despite patent inferior iridotomy and no sign of a pupillary block. The IOP peak was treated topically and systemically with IOP-lowering medication. In addition, an anterior chamber paracentesis was performed to reduce the $\mathrm{SF}_{6}$ volume within the anterior chamber. Under this treatment, IOP normalized within the first $18 \mathrm{~h}$ after surgery. Bubbles of $\mathrm{SF}_{6}$ gas could be visualized within the bleb in slit-lamp photography and optical coherence tomography (Fig. 1). Six days after surgery, the patient could be discharged to ambulatory care. On the last day of hospitalization, IOP was $12 \mathrm{~mm}$ Hg and BCVA was 0.5 logMAR in the right eye. The cornea was clear with a well-attached corneal graft and a remaining $\mathrm{SF}_{6}$ volume of $30 \%$.

\section{Case 2}

In January 2018, a 77-year-old female patient was scheduled for pseudophakic DMEK surgery due to Fuchs endothelial dystrophy in the left eye. In addition, the patient presented secondary open-angle glaucoma due to pseudoexfoliation syndrome and had undergone trabeculectomy in the left eye in 2011. On admission to the hospital, the patient's left eye had an IOP of $6 \mathrm{~mm} \mathrm{Hg}$ without any topical or systemic IOP-lowering medication. Approximately $6 \mathrm{~h}$ after an uneventful DMEK procedure - performed by an experienced surgeon (C.C.) in a standardized fashion [16] - IOP was $32 \mathrm{~mm} \mathrm{Hg}$ in the left eye. The patient was given $250 \mathrm{mg}$ of acetazolamide systemically to lower aqueous humor production. Two hours later, IOP had increased to $42 \mathrm{~mm} \mathrm{Hg}$. Slit-lamp examination showed a deep anterior chamber with no sign of pupillary block and a functional inferior iridotomy. Bubbles of $\mathrm{SF}_{6}$ were present inside the trabeculectomy bleb. Cautious anterior chamber paracentesis was performed to lower the IOP to $10 \mathrm{~mm} \mathrm{Hg}$. The remaining volume of $\mathrm{SF}_{6}$ was at $50 \%$. In the following days, the patient was given $250 \mathrm{mg}$ acetazolamide t.i.d. and potassium substitution as well as pilocarpine $2 \%$ t.i.d. as part of the postoperative standard medication after DMEK [11]. With this treatment, no further IOP peak could be observed. In every slit-lamp examination up to day 4 after DMEK, gas filling of the bleb was observed (Fig. 2). On postoperative day 6, a rebubbling procedure was recommended because of focal graft detachment. Eight days after surgery, the patient could be discharged from the hospital to ambulatory care. On the last day of hospitalization, IOP was $10 \mathrm{~mm} \mathrm{Hg}$ and BCVA was $0.8 \operatorname{logMAR}$ in the left eye. The cornea was clear with a wellattached corneal graft, a remaining $\mathrm{SF}_{6}$ volume of $40 \%$, and gas filling inside the bleb of approximately $80 \%$.

\section{Discussion}

The two cases presented in our paper describe patients with an acute postoperative IOP peak after DMEK surgery due to $\mathrm{SF}_{6}$ gas obstruction of the filtering bleb without any sign of pupillary blockage. This phenomenon is an important, newly reported complication of DMEK surgery. The eyes of both patients showed gas filling of a previously functional bleb after trabeculectomy. We hypothesize that obstruction of a functional bleb by dislocation of the gas tamponade can lead to an acute temporary insufficiency of the bleb and to a postoperative IOP peak after DMEK surgery.

The importance of DMEK to treat endothelial disorders is growing rapidly worldwide due to favorable outcomes over competing techniques $[2,17]$. Therefore, the question of the best anterior chamber tamponade to support attachment of the DMEK lamella has been in focus in 


\section{Case Reports in Ophthalmology}

the last years [1]. Potential side effects of the different types of tamponades were investigated. It is also controversial how long the tamponade should be left in the anterior chamber. Many surgeons prefer a tamponade release after a short period of complete anterior chamber filling to mitigate the risk of acute IOP elevations [7-10]. Stanzel et al. [10] reported on an intensified postoperative IOP observation within $24 \mathrm{~h}$ and found mostly mild IOP increases in 40 cases with retained tamponade. The authors concluded that adequate inferior iridotomy greatly alleviated the risk and severity of acute IOP rises after nearly full anterior chamber air tamponade in DMEK. Heinzelmann et al. [18] recently showed that postoperative IOP dips might be associated with graft detachment after DMEK. Fajgenbaum and Hollick [19] found increased IOP levels $>30 \mathrm{~mm} \mathrm{Hg}$ in $38 \%$ of patients $2 \mathrm{~h}$ after DMEK leading to a manual release of anterior chamber tamponade. As part of our group, Schaub et al. [1] found postoperative IOP decompensation necessitating treatment in $7.6 \%$ of cases receiving an $\mathrm{SF}_{6}$ tamponade $(n=105)$. In these cases, a pupillary block was the main cause for the IOP peak and anterior chamber paracentesis was performed. Steroid response is another possible reason for an IOP increase during postoperative follow-up. However, this etiology is considered unlikely in the presented cases because of three different reasons: the early timing of the IOP peak, a lack of history regarding steroid response in both patients undergoing previous glaucoma surgery, as well as the singularity of the IOP peak despite continued application of steroids.

Bleb failure leading to insufficient lowering of IOP represents a more frequent complication after trabeculectomy and can happen in early as well as later follow-up [20,21]. Frequent reasons for an early insufficiency of the bleb can be obstruction of the internal ostium of the trabeculectomy, episcleral and conjunctival fibrosis, or encapsulation of the bleb [22]. The main reasons for late failure are fibrosis and encapsulation [22]. Invasion of choroidal or conjunctival melanoma is another rare reason causing bleb insufficiency [23]. Wimmersberger et al. [24] reported on occlusion of a glaucoma drainage device after injection of $\mathrm{C}_{3} \mathrm{~F}_{8}$ gas in order to treat Descemet membrane detachment. In a case series on patients receiving Descemet stripping automated endothelial keratoplasty after previous trabeculectomy, Hardin Sheales et al. [25] found evidence for early postoperative IOP peaking suggesting a comparable mechanism.

In summary, we describe here the observed effect of a dislocation of $\mathrm{SF}_{6}$ gas tamponade into a trabeculectomy bleb after DMEK. This dislocation of the tamponade results in obstruction and acute temporary bleb insufficiency, leading to postoperative IOP peak without pupillary block. Another requirement for this mechanism seems to be a well-functioning bleb prior to DMEK surgery with controlled IOP. In contrast to a pupillary block, acute bleb blockage cannot be antagonized by preoperative iridotomy and needs to be taken into account for every glaucoma patient with functional bleb undergoing DMEK surgery with prolonged air or gas tamponade. Patients at risk should be monitored very closely to detect and treat any IOP peak within the early postoperative phase.

\section{Acknowledgments}

We thank all technical experts of our imaging laboratory and FOR 2240 "(Lymph-)Angiogenesis and Cellular Immunity in Inflammatory Diseases of the Eye" (www.for2240.de) for their support. 


\section{Statement of Ethics}

All personally identifiable information was removed. The authors have no ethical conflict to disclose.

\section{Disclosure Statement}

The authors declare no financial or competing interests or relationships. This work received no funding.

\section{References}

1 Schaub F, Enders P, Snijders K, Schrittenlocher S, Siebelmann S, Heindl LM, et al. One-year outcome after Descemet membrane endothelial keratoplasty (DMEK) comparing sulfur hexafluoride (SF6) $20 \%$ versus 100\% air for anterior chamber tamponade. Br J Ophthalmol. 2017 Jul;101(7):902-8.

2 Price DA, Kelley M, Price FW Jr, Price MO. Five-Year Graft Survival of Descemet Membrane Endothelial Keratoplasty (EK) versus Descemet Stripping EK and the Effect of Donor Sex Matching. Ophthalmology. 2018 Oct;125(10):1508-14.

3 Price MO, Calhoun P, Kollman C, Price FW Jr, Lass JH. Descemet Stripping Endothelial Keratoplasty: Ten-Year Endothelial Cell Loss Compared with Penetrating Keratoplasty. Ophthalmology. 2016 Jul;123(7):1421-7.

4 Maier P, Reinhard T, Cursiefen C. Descemet stripping endothelial keratoplasty - rapid recovery of visual acuity. Dtsch Arztebl Int. 2013 May;110(21):365-71.

5 Price FW Jr, Price MO, Arundhati A. Descemet stripping automated endothelial keratoplasty under failed penetrating keratoplasty: how to avoid complications. Am J Ophthalmol. 2011 Feb;151(2):187-8.e2.

6 Gonzalez A, Price FW Jr, Price MO, Feng MT. Prevention and Management of Pupil Block After Descemet Membrane Endothelial Keratoplasty. Cornea. 2016 Nov;35(11):1391-5.

7 Price MO, Giebel AW, Fairchild KM, Price FW Jr. Descemet's membrane endothelial keratoplasty: prospective multicenter study of visual and refractive outcomes and endothelial survival. Ophthalmology. 2009 Dec;116(12):2361-8.

8 Kruse FE, Laaser K, Cursiefen C, Heindl LM, Schlötzer-Schrehardt U, Riss S, et al. A stepwise approach to donor preparation and insertion increases safety and outcome of Descemet membrane endothelial keratoplasty. Cornea. 2011 May;30(5):580-7.

9 Zhu Q, Dapena J, Bingham GP. Learning to throw to maximum distances: do changes in release angle and speed reflect affordances for throwing? Hum Mov Sci. 2009 Dec;28(6):708-25.

10 Stanzel TP, Ersoy L, Sansanayudh W, Felsch M, Dietlein T, Bachmann B, et al. Immediate Postoperative Intraocular Pressure Changes After Anterior Chamber Air Fill in Descemet Membrane Endothelial Keratoplasty. Cornea. 2016 Jan;35(1):14-9.

11 Hoerster R, Stanzel TP, Bachmann BO, Siebelmann S, Felsch M, Cursiefen C. Intensified topical steroids as prophylaxis for macular edema after posterior lamellar keratoplasty combined with cataract surgery. Am J Ophthalmol. 2016 Mar;163:174-9.e2.

12 Gietzelt C, Lemke J, Schaub F, Hermann MM, Dietlein TS, Cursiefen C, et al. Structural reversal of disc cupping after trabeculectomy alters Bruch membrane opening-based parameters to assess neuroretinal rim. Am J Ophthalmol. 2018 Oct;194:143-52.

13 Nguyen AH, Fatehi N, Romero P, Miraftabi A, Kim E, Morales E, et al. Observational Outcomes of Initial Trabeculectomy With Mitomycin C in Patients of African Descent vs Patients of European Descent: Five-Year Results. JAMA Ophthalmol. 2018 Oct;136(10):1106-13.

14 Mathew RG, Parvizi S, Murdoch IE. Success of trabeculectomy surgery in relation to cataract surgery: 5-year outcomes. Br J Ophthalmol. 2018 Nov:bjophthalmol-2018-312972 [Epub ahead of print].

15 Caprioli J, de Leon JM, Azarbod P, Chen A, Morales E, Nouri-Mahdavi K, et al. Trabeculectomy Can Improve Long-Term Visual Function in Glaucoma. Ophthalmology. 2016 Jan;123(1):117-28.

16 Cursiefen C, Kruse FE. [DMEK: Descemet membrane endothelial keratoplasty]. Ophthalmologe. 2010 Apr;107(4):370-6.

17 Hos D, Tuac O, Schaub F, Stanzel TP, Schrittenlocher S, Hellmich M, et al. Incidence and Clinical Course of Immune Reactions after Descemet Membrane Endothelial Keratoplasty: Retrospective Analysis of 1000 Consecutive Eyes. Ophthalmology. 2017 Apr;124(4):512-8. 
Enders et al.: Bleb Blockage by Gas Tamponade after Descemet Membrane Endothelial Keratoplasty

18 Heinzelmann S, Böhringer D, Haverkamp C, Lapp T, Eberwein P, Reinhard T, et al. Influence of Postoperative Intraocular Pressure on Graft Detachment After Descemet Membrane Endothelial Keratoplasty. Cornea. 2018 Nov;37(11):1347-50.

19 Fajgenbaum MA, Hollick EJ. Does Same-Day Postoperative Increased Intraocular Pressure Affect Endothelial Cell Density After Descemet Membrane Endothelial Keratoplasty? Cornea. 2018 Dec;37(12):1484-9.

20 Hirunpatravong P, Reza A, Romero P, Kim EA, Nouri-Mahdavi K, Law SK, et al. Same-site Trabeculectomy Revision for Failed Trabeculectomy: Outcomes and Risk Factors for Failure. Am J Ophthalmol. 2016 Oct;170: 110-8.

21 Kim EA, Law SK, Coleman AL, Nouri-Mahdavi K, Giaconi JA, Yu F, et al. Long-Term Bleb-Related Infections After Trabeculectomy: Incidence, Risk Factors, and Influence of Bleb Revision. Am J Ophthalmol. 2015 Jun;159(6):1082-91.

22 Chiseliță D, Antohi I, Neamţu N. [Filtering bleb failure of open angle glaucoma]. Oftalmologia. 2002;53(2): 81-8.

23 Tay E, Cree IA, Hungerford J, Franks W. Recurrence of treated ciliary body melanoma following trabeculectomy. Clin Exp Ophthalmol. 2009 Jul;37(5):503-5.

24 Wimmersberger Y, Bergin C, Sharkawi E. Reattachment of Descemet's membrane using C3F8 gas in an eye with a Baerveldt aqueous shunt. Klin Monatsbl Augenheilkd. 2013 Apr;230(4):363-4.

25 Hardin Sheales MP, Chan E, Ang GS, George Kong YX. Elevated Intraocular Pressure after Descemet Stripping Automated Endothelial Keratoplasty in Patients with a Trabeculectomy: A Case Series. J Curr Glaucoma Pract. 2015 Sep-Dec;9(3):100-3. 


\section{Case Reports in Ophthalmology}
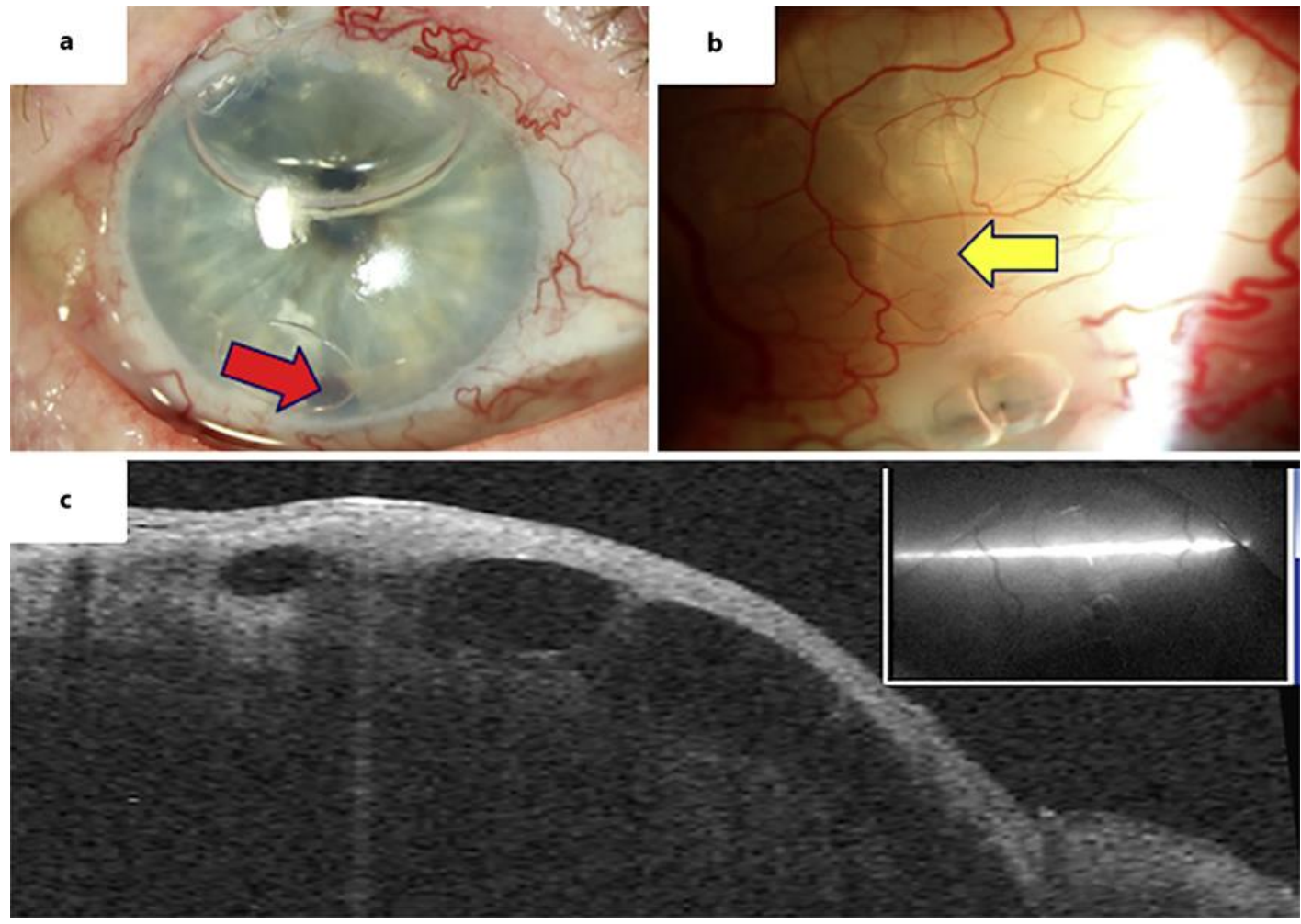

Fig. 1. Case 1. Sulfur hexafluoride gas induced acute filtering bleb failure after Descemet membrane endothelial keratoplasty. a Slit-lamp photography shows gas tamponade in the anterior chamber covering half of the pupil and inferior patent iridotomy (red arrow; note deep anterior chamber without signs of pupillary block and air bubbles around the red arrow below the contact lens). b Multiple gas bubbles visible within the bleb (yellow arrow). c Visualization of gas bubbles inside the bleb using optical coherence tomography. Intraocular pressure was elevated to $50 \mathrm{~mm} \mathrm{Hg}$.
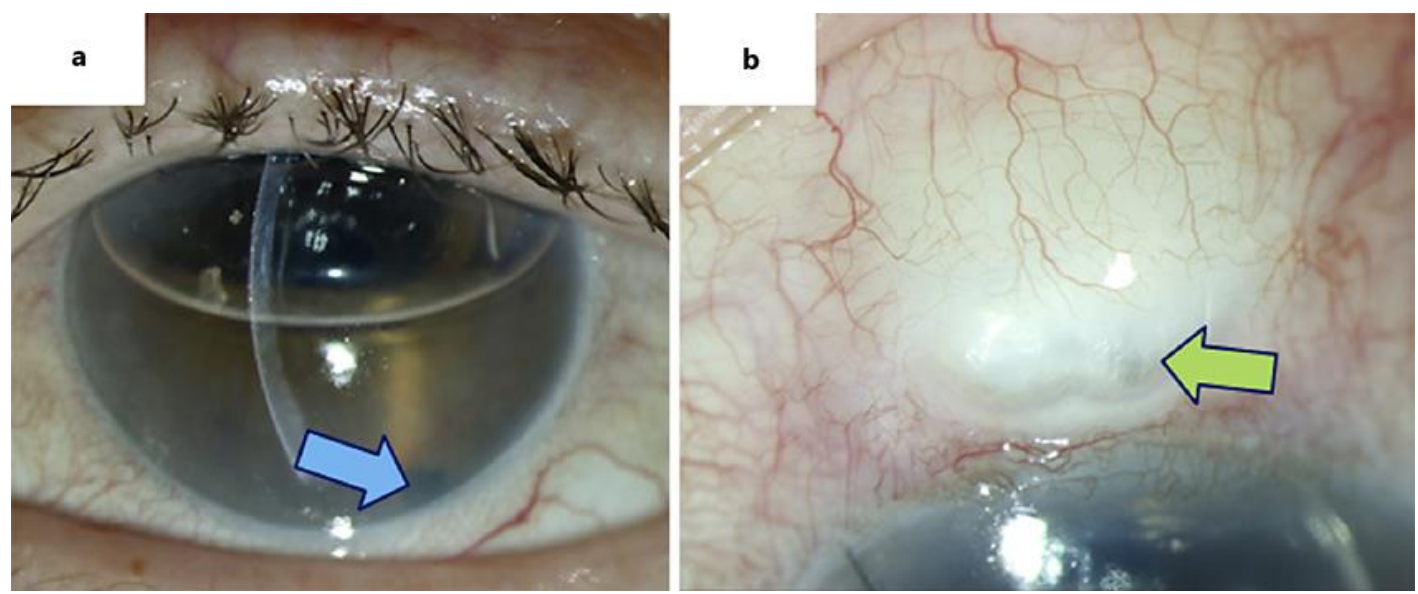

Fig. 2. Case 2. Sulfur hexafluoride gas induced acute filtering bleb failure after Descemet membrane endothelial keratoplasty. a Slit-lamp photography shows gas tamponade in the anterior chamber covering the pupil and inferior patent iridotomy (blue arrow; note deep anterior chamber). b Gas bubbles visible within the bleb (green arrow). Intraocular pressure was elevated to $50 \mathrm{~mm} \mathrm{Hg}$. 